\title{
Atomic Electron Tomography: Adding a New Dimension to See Single Atoms in Materials
}

Jianwei Miao ${ }^{1}$, Yongsoo Yang ${ }^{1}$, Jihan Zhou ${ }^{1}$, Xuezeng Tian ${ }^{1}$, Yao Yang ${ }^{1}$, Dennis S. Kim ${ }^{1}$, Alan Pryor Jr. ${ }^{1}$, Peter Ercius ${ }^{2}$, Colin Ophus ${ }^{2}$, M. C. Scott ${ }^{2,3}$, Chien-Chun Chen ${ }^{4}$, Wolfgang Theis ${ }^{5}$, Markus Eisenbach $^{6}$, Paul R. C. Kent ${ }^{7,8}$, Renat F. Sabirianov ${ }^{9}$ and Hao Zeng ${ }^{10}$

1. Department of Physics \& Astronomy and California NanoSystems Institute, and University of California, Los Angeles, CA.

2. National Center for Electron Microscopy, Molecular Foundry, Lawrence Berkeley National Laboratory, Berkeley, CA.

3. Department of Materials Science and Engineering, University of California, Berkeley, CA.

4. Department of Physics, National Sun Yat-sen University, Kaohsiung, Taiwan.

5. Nanoscale Physics Research Laboratory, School of Physics and Astronomy, University of Birmingham, Birmingham, UK.

6. National Center for Computational Sciences, Oak Ridge National Laboratory, Oak Ridge, TN.

${ }^{7}$ Computer Science and Mathematics Division, Oak Ridge National Laboratory, Oak Ridge, TN

${ }^{8 .}$ Center for Nanophase Materials Sciences, Oak Ridge National Laboratory, Oak Ridge, TN.

9. Department of Physics, University of Nebraska at Omaha, Omaha, NE.

10. Department of Physics, University at Buffalo, the State University of New York, Buffalo, NY.

To understand material properties and functionality at the fundamental level, one must know the 3D positions of atoms with high precision. For crystalline materials, crystallography has provided this information since the pioneering work of Max von Laue, William Henry Bragg, and William Lawrence Bragg over a century ago. However, perfect crystals are rare in nature. Real materials often contain crystal defects, surface reconstructions, nanoscale heterogeneities, and disorders, which strongly influence material properties and performance. Here, we present atomic electron tomography (AET) for 3D structure determination of crystal defects and disordered materials at the single-atom level (1). Using powerful Fourier-based iterative algorithms (2,3), we first demonstrated electron tomography at 2.4- $\AA$ resolution without assuming crystallinity in 2012 (4). We then applied AET to image the 3D structure of grain boundaries and stacking faults and the 3D core structure of edge and screw dislocations at atomic resolution (5). Furthermore, in combination of AET and atom tracing algorithms, we localized the coordinates of individual atoms and point defects in materials with a 3D precision of $\sim 19 \mathrm{pm}$, allowing direct measurements of 3D atomic displacements and the full strain tensor (6). More recently, we determined the 3D coordinates of 6,569 Fe and 16,627 Pt atoms in an FePt nanoparticle, and correlated chemical order/disorder and crystal defects with material properties at the individual atomic level (7). We identified rich structural variety with unprecedented 3D detail including atomic composition, grain boundaries, anti-phase boundaries, anti-site point defects and swap defects. We showed that the experimentally measured coordinates and chemical species with $22 \mathrm{pm}$ precision can be used as direct input for density functional theory calculations of material properties such as atomic spin and orbital magnetic moments and local magnetocrystalline anisotropy (7). Looking forward, AET will not only advance our ability in 3D atomic structure determination of crystal defects and disordered materials, but also transform our understanding of materials properties and functionality at the single atomic level [8]. 
References:

[1] Miao, J., Ercius, P. and Billinge, S. Science 353 (2016), aaf2157.

[2] Miao, J., Föster, F. \& Levi, O. Phys. Rev. B 72 (2005), p. 052103.

[3] Pryor, A. Jr. et al, Sci. Rep. 7 (2017), p. 10409.

[4] Scott, M. C. et al, Nature 483 (2012), p. 444.

[5] Chen, C.-C. et al, Nature 496 (2013), p. 74.

[6] Xu, R. et al, Nature Mater. 14 (2015), p. 1099.

[7] Yang, Y. et al, Nature 542 (2017), p. 75.

[8] This work was supported by STROBE: A National Science Foundation Science \& Technology Center under Grant No. DMR 1548924, and the Office of Basic Energy Sciences of the U.S. Department of Energy (Grant No. DE-SC0010378). ADF-STEM imaging was performed on TEAM I at the Molecular Foundry, which is supported by the Office of Science, Office of Basic Energy Sciences of the U.S. Department of Energy under Contract No. DE-AC02—05CH11231.

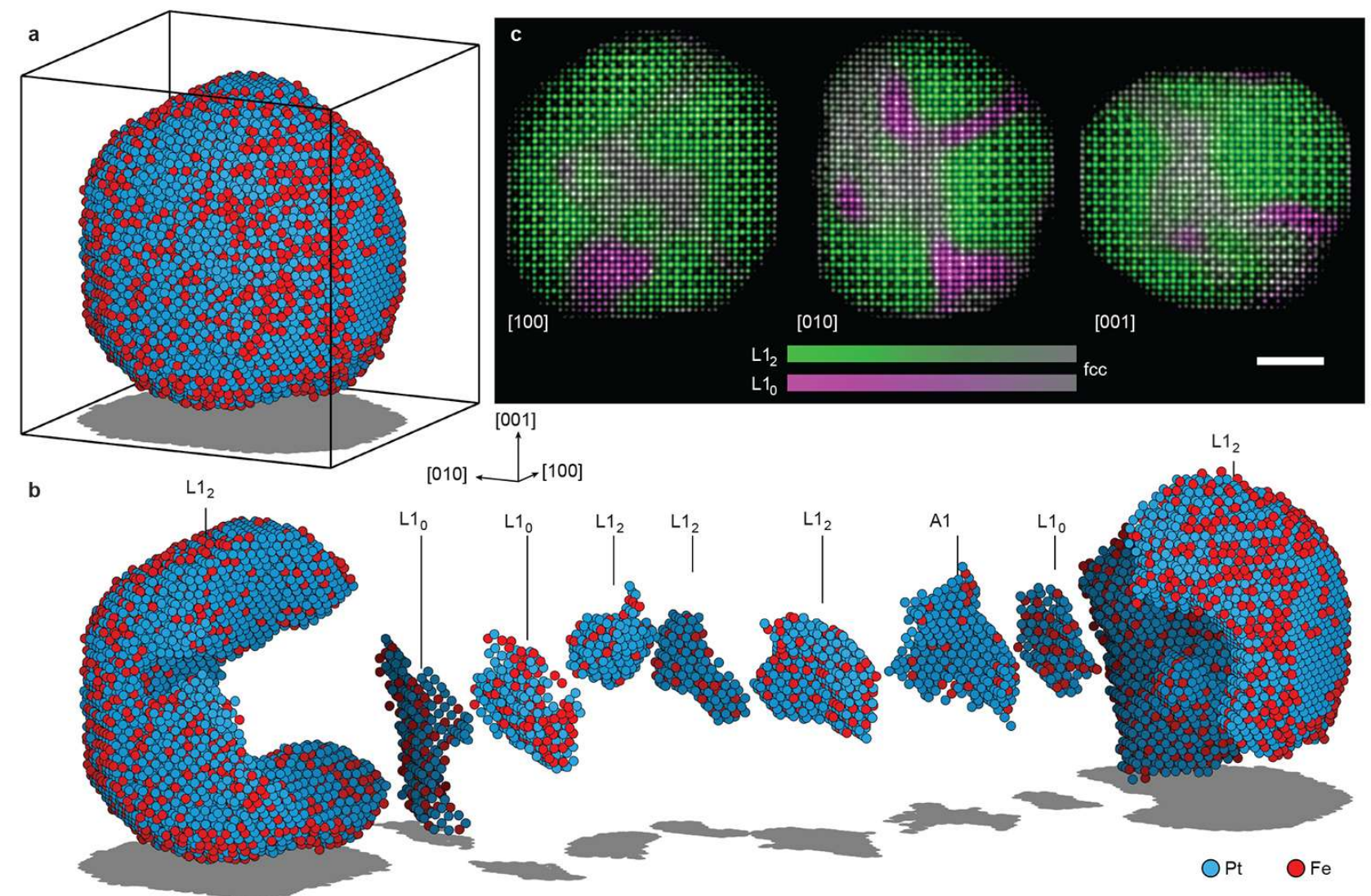

Figure 1. 3D determination of atomic coordinates and chemical order/disorder of an FePt nanoparticle. (a) Overview of the 3D positions of individual atomic species with Fe atoms in red and Pt atoms in blue. (b) The nanoparticle consists of two large L12 grains, three small L12 grains, three small L10 grains and a Pt-rich A1 grain. (c) Multislice images obtained from the experimental 3D atomic model along the [100], [010] and [001] directions, where several 'L10 grains' (magenta) appearing in the 2D images are deceptive structural information. Scale bar, $2 \mathrm{~nm}$. 